\title{
New Diterpenoids of Sigesbeckia pubescens (Makino) Makino
}

\author{
Aleksey V. Myagchilov 1,2,*(D) , Larisa I. Sokolova ${ }^{1}$, Peter G. Gorovoy ${ }^{2 f(D)}$ \\ 1 School of Natural Sciences, Far Eastern Federal University, 10 Ajax Bay, Russky Island, Vladivostok, Russia; \\ dfdfdf47@yandex.ru (A.V.M.); lisokolova@bk.ru (L.I.S.); \\ 2 G. B. Elyakov Pacific Institute of Bioorganic Chemistry, Far Eastern Branch, Russian Academy of Science, 100 let \\ Vladivostoku Prosp. 159, Vladivostok, Russia; petrgorovoy@gmail.com (P.G.G.); \\ * Correspondence: dfdfdf47@yandex.ru (A.V.M.);
}

Scopus Author ID 57194203891

Received: 14.10.2021; Revised: 20.11.2021; Accepted: 24.11.2021; Published: 5.12.2021

\begin{abstract}
The composition of diterpenoids isolated from the aerial parts of Sigesbeckia pubescens growing in Primorsky Territory was investigated. Diterpenoids $\beta$-glucopyranosyl-18-acetoxy-16 $\alpha, 17$ dihydroxykauran-19-oate (1), 15-O-malonylkirenol (2), and 16-O-malonylkirenol (3) were isolated by extraction and preparative column chromatography. The diterpenoids 1-3 were new compounds, the structures of which were established using NMR spectroscopy and mass spectrometry.
\end{abstract}

Keywords: Sigesbeckia pubescens; diterpenoids; NMR spectroscopy.

(C) 2021 by the authors. This article is an open-access article distributed under the terms and conditions of the Creative Commons Attribution (CC BY) license (https://creativecommons.org/licenses/by/4.0/).

\section{Introduction}

Sigesbeckia pubescens (Makino) Makino - an annual plant of the Asteraceae family (Compositae), is widespread on the territory of China, Korea, Japan, and the Primorsky Region of the Russian Far East. An annual plant Sigesbeckia pubescens is used in traditional Chinese medicine to treat rheumatic arthritis, hypertension, malaria, and neurasthenia [1-4]. This plant has anti-inflammatory, anti-allergic, antithrombotic, antirheumatic, antihistamine, and antiatherosclerotic properties [5-14]. The medicinal properties of Sigesbeckia pubescens are due to the presence in its composition of flavonoids, sesquiterpenoids, triterpenoids, and diterpenoids [5,7,15-19]. Pharmacological studies of this plant have shown that diterpenoids are the main biologically active compounds used to treat rheumatic arthritis [1]. It is necessary to conduct further studies of the composition of new diterpenoid compounds of this plant for further investigation of the pharmacological properties of Sigesbeckia pubescens.

\section{Materials and Methods}

\subsection{General experimental procedures.}

Mass spectrometric analysis used a Bruker MaXis (USA) with electrospray ionization (ESI) in positive-ion mode. NMR spectra were recorded in DMSO- $\mathrm{d}_{6}$ on a Bruker AVANCE400 spectrometer (USA) at an operating frequency $400 \mathrm{MHz}$. 


\subsection{Plant material.}

The aerial parts of Sigesbeckia pubescens were collected in Primorsky Territory (in the vicinity of Shkotovo village, Shkotovo District, September 2020, during flowering) and identified by professor Peter G. Gorovoy (G. B. Elyakov Pacific Institute of Bioorganic Chemistry FEB RAS). A voucher specimen (№ 103618) was deposited in the Pacific Institute of Bioorganic Chemistry FEB RAS.

\subsection{Extraction and isolation}

The aerial parts of Sigesbeckia pubescens $(100 \mathrm{~g})$ were extracted with refluxing EtOH $(70 \%, 1000 \mathrm{ml})$ on a boiling-water bath for $1.5 \mathrm{~h}$. The resulting extract was concentrated in a rotary evaporator to an aqueous residue that was worked up successively with $\mathrm{CCl}_{4}, \mathrm{EtOAc}$, and $n-\mathrm{BuOH}$.

The $\mathrm{BuOH}$ extract was dried over $\mathrm{Na}_{2} \mathrm{SO}_{4}$, filtered, and evaporated to dryness at reduced pressure. The dry residue $(15 \mathrm{~g})$ was treated with $\mathrm{EtOH}(96 \%, 10 \mathrm{ml})$ and mixed with silica gel (5g, 70-230 mesh fraction). The mixture of extract and silica gel was dried at room temperature, transferred to a column $(1 \times 15 \mathrm{~cm})$ of silica gel $(70-230 \mathrm{mesh})$, and eluted with $\mathrm{CCl}_{4}-\mathrm{EtOH}$ (EtOH content increasing from 0 to $100 \%$ ). As a result of the $\mathrm{BuOH}$ extract of Sigesbeckia pubescens identified the compounds $\mathbf{1}$ (12 mg), 2 (35 mg), and $\mathbf{3}$ (28 mg).

$\beta$-glucopyranosyl-18-acetoxy-16 $\alpha, 17$-dihydroxykauran-19-oate (1)

HR-ESI-MS $m / z: 579.2807[\mathrm{M}+\mathrm{Na}]^{+}$(calcd. for $\mathrm{C}_{28} \mathrm{H}_{44} \mathrm{O}_{11} \mathrm{Na}^{+}, 579.2776$ ); ${ }^{1} \mathrm{H}$ NMR and ${ }^{13} \mathrm{C}$ NMR: Table 1.

15-O-malonylkirenol (2)

HR-ESI-MS $m / z$ : $447.5169[\mathrm{M}+\mathrm{Na}]^{+}$(calcd. for $\mathrm{C}_{23} \mathrm{H}_{36} \mathrm{O}_{7} \mathrm{Na}^{+}, 447.5175$ ); ${ }^{1} \mathrm{H} \mathrm{NMR}$ and ${ }^{13} \mathrm{C}$ NMR: Table 1.

16-O-malonylkirenol (3)

HR-ESI-MS $m / z: 447.5170[\mathrm{M}+\mathrm{Na}]^{+}$(calcd. for $\mathrm{C}_{23} \mathrm{H}_{36} \mathrm{O}_{7} \mathrm{Na}^{+}, 447.5175$ ); ${ }^{1} \mathrm{H}$ NMR and ${ }^{13} \mathrm{C}$ NMR: Table 1.

\section{Results and Discussion}

Preparative column chromatography on the butanol extract of Sigesbeckia pubescens afforded the three new diterpenoids (Figure 1).

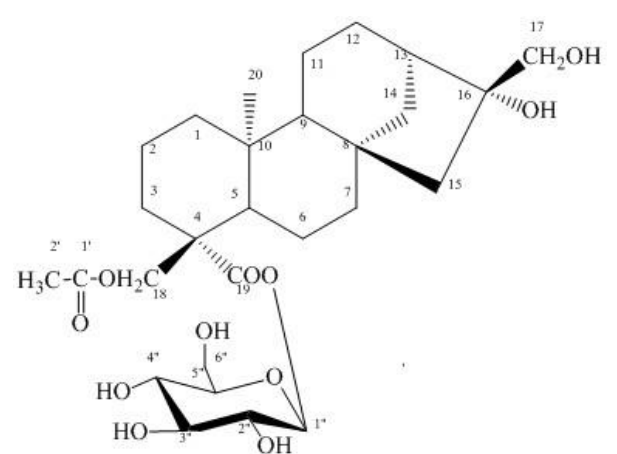

1

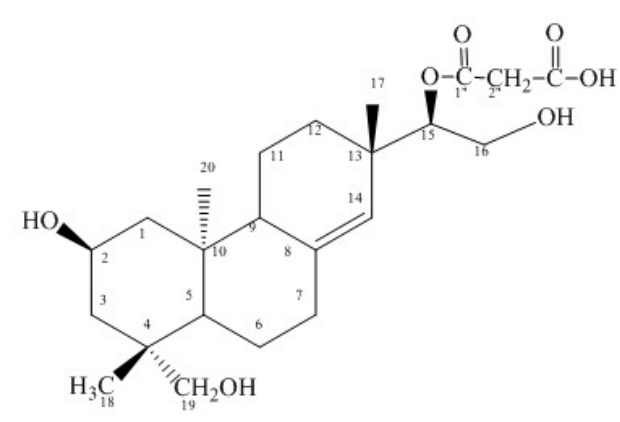

2 


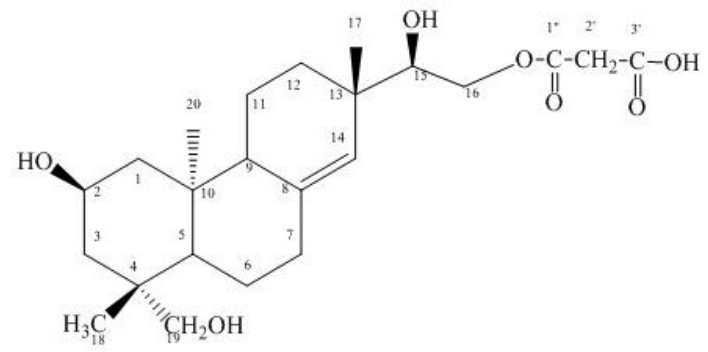

3

Figure 1. Chemical structures of diterpenoids 1-3.

Table 1. ${ }^{1} \mathrm{H}$ and ${ }^{13} \mathrm{C}$ NMR data of $1-3$ in $\mathrm{d}^{6}$-DMSO $(\delta$ in ppm, $\mathrm{J} / \mathrm{Hz}$ ).

\begin{tabular}{|c|c|c|c|c|c|c|c|c|c|c|c|}
\hline № & & ${ }^{1} \mathbf{H}$ & ${ }^{13} \mathrm{C}$ & № & & ${ }^{1} \mathbf{H}$ & ${ }^{13} \mathrm{C}$ & № & & ${ }^{1} \mathbf{H}$ & ${ }^{13} \mathrm{C}$ \\
\hline & & 1 & & & & 2 & & & & 3 & \\
\hline 1 & $\begin{array}{l}\mathrm{a} \\
\mathrm{b}\end{array}$ & $\begin{array}{l}0.68(\mathrm{~m}) \\
1.71(\mathrm{~m}) \\
\end{array}$ & 40.1 & 1 & $\begin{array}{l}\mathrm{a} \\
\mathrm{b}\end{array}$ & $\begin{array}{l}0.84(\mathrm{~m}) \\
1.78(\mathrm{~m}) \\
\end{array}$ & 48.6 & 1 & $\begin{array}{l}\mathrm{a} \\
\mathrm{b}\end{array}$ & $\begin{array}{l}0.86(\mathrm{~m}) \\
1.78(\mathrm{~m}) \\
\end{array}$ & 48.7 \\
\hline 2 & $\begin{array}{l}\mathrm{a} \\
\mathrm{b}\end{array}$ & $\begin{array}{l}1.35(\mathrm{~m}) \\
1.83(\mathrm{~m})\end{array}$ & 18.4 & 2 & & $3.55(\mathrm{~m})$ & 62.9 & 2 & & $3.56(\mathrm{~m})$ & 63.0 \\
\hline 3 & $\begin{array}{l}\mathrm{a} \\
\mathrm{b}\end{array}$ & $\begin{array}{l}1.04(\mathrm{~m}) \\
2.17(\mathrm{~m})\end{array}$ & 32.5 & 3 & $\begin{array}{l}\mathrm{a} \\
\mathrm{b}\end{array}$ & $\begin{array}{l}0.69(\mathrm{~m}) \\
2.04(\mathrm{~m})\end{array}$ & 44.8 & 3 & $\begin{array}{l}\mathrm{a} \\
\mathrm{b}\end{array}$ & $\begin{array}{l}0.69(\mathrm{~m}) \\
2.04(\mathrm{~m})\end{array}$ & 45.0 \\
\hline 4 & & - & 47.7 & 4 & & - & 40.3 & 4 & & - & 40.3 \\
\hline 5 & & $1.25(\mathrm{~m})$ & 51.3 & 5 & & $1.07(\mathrm{~m})$ & 55.0 & 5 & & $1.07(\mathrm{~m})$ & 55.1 \\
\hline 6 & $\begin{array}{l}\mathrm{a} \\
\mathrm{b}\end{array}$ & $\begin{array}{l}1.55(\mathrm{~m}) \\
1.77(\mathrm{~m})\end{array}$ & 21.8 & 6 & $\begin{array}{l}\mathrm{a} \\
\mathrm{b}\end{array}$ & $\begin{array}{l}1.20(\mathrm{~m}) \\
1.58(\mathrm{~m})\end{array}$ & 22.3 & 6 & $\begin{array}{l}\mathrm{a} \\
\mathrm{b}\end{array}$ & $\begin{array}{l}1.19(\mathrm{~m}) \\
1.59(\mathrm{~m})\end{array}$ & 22.3 \\
\hline 7 & $\begin{array}{l}\mathrm{a} \\
\mathrm{b}\end{array}$ & $\begin{array}{l}1.30(\mathrm{~m}) \\
1.43(\mathrm{~m}) \\
\end{array}$ & 41.9 & 7 & $\begin{array}{l}\mathrm{a} \\
\mathrm{b}\end{array}$ & $\begin{array}{l}1.90(\mathrm{~m}) \\
2.22(\mathrm{~m}) \\
\end{array}$ & 36.2 & 7 & $\begin{array}{l}\mathrm{a} \\
\mathrm{b}\end{array}$ & $\begin{array}{l}1.90(\mathrm{~m}) \\
2.20(\mathrm{~m}) \\
\end{array}$ & 36.3 \\
\hline 8 & & - & 44.2 & 8 & & - & 139.2 & 8 & & - & 138.3 \\
\hline 9 & & $0.92(\mathrm{~m})$ & 55.8 & 9 & & $1.69(\mathrm{~m})$ & 50.6 & 9 & & $1.69(\mathrm{~m})$ & 50.9 \\
\hline 10 & & - & 39.4 & 10 & & - & 39.4 & 10 & & - & 39.4 \\
\hline 11 & $\begin{array}{l}\mathrm{a} \\
\mathrm{b}\end{array}$ & $\begin{array}{l}1.47(\mathrm{~m}) \\
1.79(\mathrm{~m}) \\
\end{array}$ & 18.6 & 11 & $\begin{array}{l}\mathrm{a} \\
\mathrm{b}\end{array}$ & $\begin{array}{l}1.41(\mathrm{~m}) \\
1.41(\mathrm{~m})\end{array}$ & 18.6 & 11 & $\begin{array}{l}\mathrm{a} \\
\mathrm{b}\end{array}$ & $\begin{array}{l}1.41(\mathrm{~m}) \\
1.41(\mathrm{~m})\end{array}$ & 18.6 \\
\hline 12 & $\begin{array}{l}\mathrm{a} \\
\mathrm{b}\end{array}$ & $\begin{array}{l}1.26(\mathrm{~m}) \\
1.46(\mathrm{~m})\end{array}$ & 26.2 & 12 & $\begin{array}{l}\mathrm{a} \\
\mathrm{b}\end{array}$ & $\begin{array}{l}0.81(\mathrm{~m}) \\
1.91(\mathrm{~m})\end{array}$ & 32.3 & 12 & $\begin{array}{l}\mathrm{a} \\
\mathrm{b}\end{array}$ & $\begin{array}{l}0.81(\mathrm{~m}) \\
1.90(\mathrm{~m})\end{array}$ & 32.1 \\
\hline 13 & & $1.82(\mathrm{~m})$ & 44.9 & 13 & & - & 37.0 & 13 & & - & 37.6 \\
\hline 14 & $\begin{array}{l}\mathrm{a} \\
\mathrm{b}\end{array}$ & $\begin{array}{l}1.46(\mathrm{~m}) \\
1.71(\mathrm{~m})\end{array}$ & 37.0 & 14 & & $5.13(\mathrm{~s})$ & 127.6 & 14 & & $5.15(\mathrm{~s})$ & 128.8 \\
\hline 15 & $\begin{array}{l}\mathrm{a} \\
\mathrm{b}\end{array}$ & $\begin{array}{l}1.25(\mathrm{~m}) \\
1.39(\mathrm{~m})\end{array}$ & 53.1 & 15 & & $4.84(\mathrm{dd}, 6.6,2.1)$ & 79.1 & 15 & & $3.51(\mathrm{~m})$ & 71.9 \\
\hline 16 & & - & 81.0 & 16 & $\begin{array}{l}\mathrm{a} \\
\mathrm{b}\end{array}$ & $\begin{array}{l}3.44(\mathrm{~m}) \\
3.56(\mathrm{~m})\end{array}$ & 60.7 & 16 & $\begin{array}{l}\mathrm{a} \\
\mathrm{b}\end{array}$ & $\begin{array}{l}4.09(\mathrm{dd}, 8.8,2.2) \\
4.11(\mathrm{dd}, 8.8,2.1)\end{array}$ & 67.1 \\
\hline 17 & $\begin{array}{l}\mathrm{a} \\
\mathrm{b}\end{array}$ & $\begin{array}{l}3.38(\mathrm{~m}) \\
3.46(\mathrm{~d}, 11.6)\end{array}$ & 65.7 & 17 & & $0.83(\mathrm{~s}, 3 \mathrm{H})$ & 23.5 & 17 & & $0.79(\mathrm{~s}, 3 \mathrm{H})$ & 22.8 \\
\hline 18 & $\begin{array}{l}\mathrm{a} \\
\mathrm{b}\end{array}$ & $\begin{array}{l}3.82(\mathrm{~d}, 10.4) \\
4.26(\mathrm{~d}, 10.4)\end{array}$ & 70.6 & 18 & & $0.87(\mathrm{~s}, 3 \mathrm{H})$ & 28.1 & 18 & & $0.87(\mathrm{~s}, 3 \mathrm{H})$ & 28.1 \\
\hline 19 & & - & 173.4 & 19 & $\begin{array}{l}\mathrm{a} \\
\mathrm{b}\end{array}$ & $\begin{array}{l}3.11(\mathrm{~d}, 10) \\
3.43(\mathrm{~d}, 10)\end{array}$ & 63.9 & 19 & $\begin{array}{l}\mathrm{a} \\
\mathrm{b}\end{array}$ & $\begin{array}{l}3.12(\mathrm{~d}, 10) \\
3.44(\mathrm{~d}, 10)\end{array}$ & 63.9 \\
\hline 20 & & $0.84(\mathrm{~s}, 3 \mathrm{H})$ & 15.6 & 20 & & $0.72(\mathrm{~s}, 3 \mathrm{H})$ & 16.7 & 20 & & $0.67(\mathrm{~s}, 3 \mathrm{H})$ & 16.8 \\
\hline $1^{\prime}$ & & - & 170.8 & $1^{\prime}$ & & - & 167.3 & $\mathbf{1}^{\prime}$ & & - & 167.5 \\
\hline $2 \prime$ & & $1.94(\mathrm{~s}, 3 \mathrm{H})$ & 20.9 & $2 \prime$ & & $3.31(\mathrm{~s}, 2 \mathrm{H})$ & 42.6 & $2 \prime$ & & $3.31(\mathrm{~s}, 2 \mathrm{H})$ & 42.2 \\
\hline 1" & & $5.29(\mathrm{~d}, 8.2)$ & 94.5 & $3 \prime$ & & - & 168.7 & $3^{\prime}$ & & - & 168.5 \\
\hline $2^{\prime \prime}$ & & $3.11(\mathrm{~m})$ & 72.9 & & & & & & & & \\
\hline $3^{\prime \prime}$ & & $3.15(\mathrm{~m})$ & 77.2 & & & & & & & & \\
\hline $4^{\prime \prime}$ & & $3.10(\mathrm{~m})$ & 70.1 & & & & & & & & \\
\hline $5^{\prime \prime}$ & & $3.21(\mathrm{~m})$ & 78.1 & & & & & & & & \\
\hline $6^{\prime \prime}$ & $\begin{array}{l}\mathrm{a} \\
\mathrm{b}\end{array}$ & $\begin{array}{l}3.40(\mathrm{~m}) \\
3.61(\mathrm{~d}, 10.4)\end{array}$ & 61.2 & & & & & & & & \\
\hline
\end{tabular}

\subsection{Compound 1.}

The molecular formula was identified as $\mathrm{C}_{28} \mathrm{H}_{44} \mathrm{O}_{11}$ by HR-ESI-MS data ([M+Na] $]^{+} \mathrm{m} / \mathrm{z}$ 579.2807, calcd. 579.2776). 
The ${ }^{1} \mathrm{H}$ NMR spectrum (Table 1) displayed signals for an anomeric proton at $\delta_{\mathrm{H}} 5.29$ (d, H-1", J=8.2 Hz), two methyl groups at $\delta_{\mathrm{H}} 0.84\left(\mathrm{~s}, \mathrm{CH}_{3}-20\right), 1.94\left(\mathrm{~s}, \mathrm{CH}_{3}-2^{\prime}\right)$. The ${ }^{13} \mathrm{C}$ NMR spectrum revealed two methyls, twelve methylenes (three oxygenated, at $\delta_{\mathrm{C}} 65.7$ and 70.6 in the aglycone, one oxygenated carbon in a sugar moiety at $\delta_{\mathrm{C}} 61.2$ ), eight methines, and six quaternary carbons (two carbonyl carbon at $\delta_{\mathrm{C}} 170.8$ and 173.4). The HMBC correlations from $\mathrm{CH}_{3}-20$ to C-1, C-6, and C-10; from $\mathrm{CH}_{2}-17$ to C-16; from $\mathrm{CH}_{2}-18$ to C-3, C-4, and C-19, as depicted in Figure 2. The relative configuration has established the correlations in the NOESY spectrum of $\mathrm{CH}_{2}-18 / \mathrm{H}-5 / \mathrm{H}-9$ and $\mathrm{CH}_{2}-17 / \mathrm{H}-9$ (Figure 2). The NMR spectra of 1 were similar to those of $16 \alpha, 17,18$-trihydroxykauran-19-oic acid [20] except for an additional acetyl moiety $\left[\delta_{\mathrm{H}} 1.94(3 \mathrm{H}, \mathrm{s}) ; \delta_{\mathrm{C}} 170.8,20.9\right]$ and glucopyranosyl moiety $\left(\delta_{\mathrm{C}} 72.9,77.2,70.1,78.1\right.$, and 61.2). The acetyl group was attached to $\mathrm{C}-18$, as was supported by an HMBC cross-peaks between $\mathrm{H}_{2}-18\left(\delta_{\mathrm{H}} 3.82\right.$ and 4.26) and the carboxyl carbon (173.4). The coupling constant of the anomeric proton at $\delta_{\mathrm{H}} 5.29(\mathrm{~d}, \mathrm{~J}=8.2 \mathrm{~Hz})$ and $\mathrm{C}-1^{\prime \prime}$ signal at $\delta_{\mathrm{C}} 94.5$ indicated a $\beta$ glucopyranose. In the HMBC spectrum (Figure 2), the correlation between the anomeric proton $\mathrm{H}-1$ " $\left(\delta_{\mathrm{H}} 5.29\right)$ and $\mathrm{C}-19\left(\delta_{\mathrm{C}} 173.4\right)$ showed that sugar moiety in diterpenoid was located at $\mathrm{C}$ 19. Therefore, the structure of compound $\mathbf{3}$ was defined as $\beta$-glucopyranosyl-18-acetoxy$16 \alpha, 17$-dihydroxykauran-19-oate.

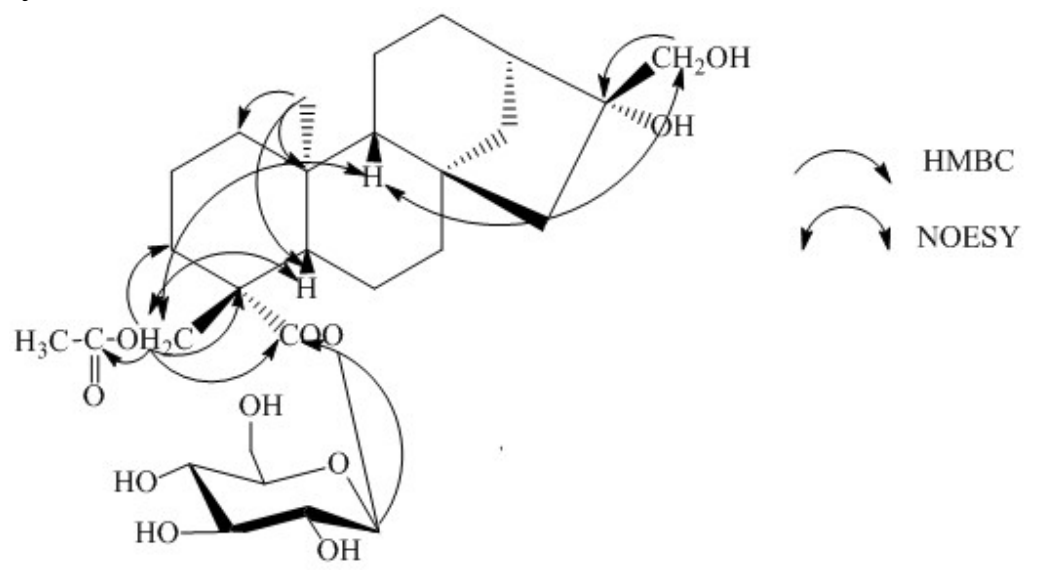

Figure 2. Key HMBC and NOESY correlations of compound 1.

\subsection{Compound 2.}

The molecular formula was identified as $\mathrm{C}_{23} \mathrm{H}_{36} \mathrm{O}_{7}$ by HR-ESI-MS $\left([\mathrm{M}+\mathrm{Na}]^{+} \mathrm{m} / \mathrm{z}\right.$ 447.5169, calcd. 447.5175).

The ${ }^{1} \mathrm{H}$ NMR spectrum (Table 1) displayed signals for an olefinic proton at $\delta_{\mathrm{H}} 5.13(\mathrm{~s}$, 14-H), three methyl groups at $\delta_{\mathrm{H}} 0.83\left(\mathrm{~s}, \mathrm{CH}_{3}-17\right), 0.87\left(\mathrm{~s}, \mathrm{CH}_{3}-18\right)$ and $0.72\left(\mathrm{~s}, \mathrm{CH}_{3}-20\right)$. The ${ }^{13} \mathrm{C}$ NMR spectrum revealed three methyls, nine methylenes, four methines (including one olefinic carbon at $\delta_{\mathrm{c}}$ 127.6), and four quaternary carbons (including one olefinic carbon at $\delta_{\mathrm{c}}$ 139.2). The HMBC correlations from $\mathrm{CH}_{3}-17$ to $\mathrm{C}-12$, $\mathrm{C}-13$, and $\mathrm{C}-15$; from $\mathrm{CH}_{3}-18$ to $\mathrm{C}-3$, C-4, and C-5; from $\mathrm{CH}_{3}-20$ to C-1, C-5, C-9, and C-10; from $\mathrm{H}-14$ to $\mathrm{C}-12$ and C-13, as depicted in Figure 3. The relative configuration has established the correlations in the NOESY spectrum of $\mathrm{CH}_{3}-18 / \mathrm{H}-5 / \mathrm{H}-9, \mathrm{CH}_{3}-17 / \mathrm{H}-9$, and $\mathrm{CH}_{3}-20 / \mathrm{H}-2$ (Figure 3). The NMR data were very similar to those of kirenol [21-23] except for a set of resonances due to a malonyl moiety $\left[\delta_{\mathrm{H}} 3.31(2 \mathrm{H}, \mathrm{s}) ; \delta_{\mathrm{c}} 167.3,168.7,42.3\right]$. Availability of correlation between $\mathrm{H}-15\left(\delta_{\mathrm{H}} 4.84\right)$ and $\mathrm{C}-1^{\prime}\left(\delta_{\mathrm{c}} 167.3\right)$ in spectrum HMBC states that malonic acid moiety in diterpenoid is linked at $\mathrm{C}-15$ (Figure 3). Alkaline hydrolysis of compound $2\left(5 \% \mathrm{KOH} / \mathrm{CH}_{3} \mathrm{OH}, 100{ }^{\circ} \mathrm{C}, 60 \mathrm{~min}\right)$ 
formed the kirenol, and spectrum ${ }^{13} \mathrm{C}$ NMR displayed a diagnostic resonance at $\delta_{\mathrm{c}} 76.4$ (C-15) defined the configuration of kirenol as the $R$. There is an empirical data for the chemical shift of C-15 in ent-pimarane skeleton. The chemical shift of C-15 for $R$-configuration is $77 \mathrm{ppm}$, while a change to $79 \mathrm{ppm}$ for $S$-configuration [21,24,25]. Thus, the structure of compound 2 was determined to be $15 R$-malonyl-2 $\alpha, 16,19$-trihydroxypimar-8(14)ene (15-Omalonylkirenol).

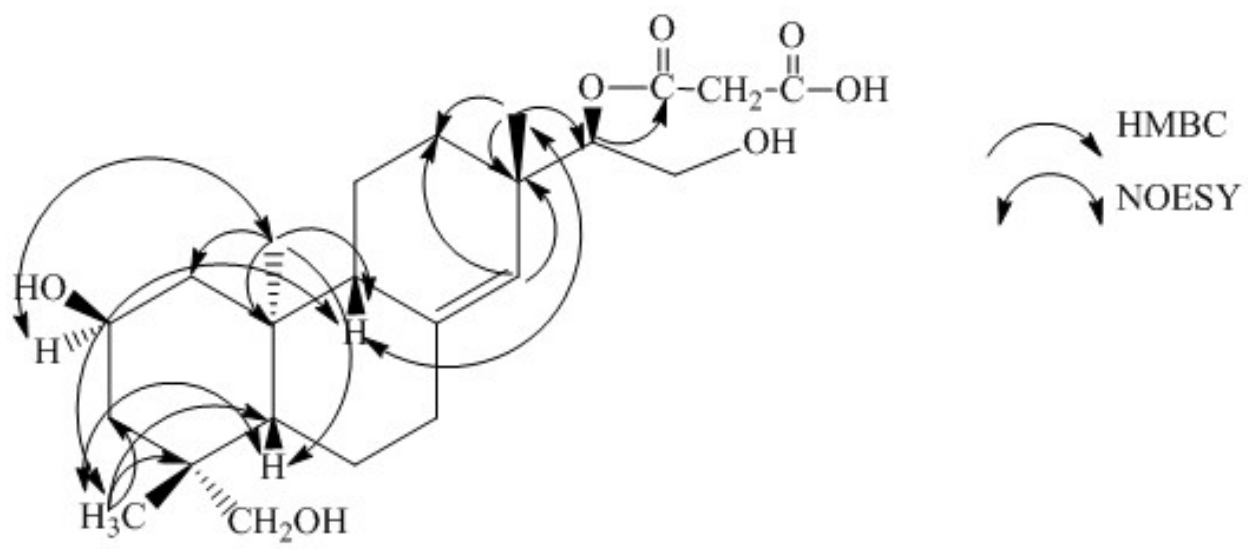

Figure 3. Key HMBC and NOESY correlations of compound 2.

\subsection{Compound 3.}

The molecular formula was established as $\mathrm{C}_{23} \mathrm{H}_{36} \mathrm{O}_{7}$ by HR-ESI-MS data $\left([\mathrm{M}+\mathrm{Na}]^{+} \mathrm{m} / z\right.$ 447.5170, calcd. 447.5175).

The ${ }^{1} \mathrm{H}$ and ${ }^{13} \mathrm{C}$ NMR spectra of compound 3 were nearly superimposable with those of compound 2 revealed a downfield shift of the signal of C-16 (+4.6 ppm) and upfield shift of the signals of $\mathrm{C}-15(-7.2 \mathrm{ppm})$, showing that $\mathrm{OH}$ group has been attached to $\mathrm{C}-16$ in diterpenoid by malonic acid $\left[\delta_{\mathrm{H}} 3.31(\mathrm{~s}, 2 \mathrm{H}) ; \delta_{\mathrm{c}} 167.5,168.5,42.2\right]$. It was also supported by an HMBC cross-peak between the $\mathrm{H}_{2}-16\left(\delta_{\mathrm{H}} 4.09\right.$ and 4.11$)$ and the carbonyl carbon $\left(\delta_{\mathrm{C}} 167.5\right)$. Alkaline hydrolysis of compound $3\left(5 \% \mathrm{KOH} / \mathrm{CH}_{3} \mathrm{OH}, 100{ }^{\circ} \mathrm{C}, 60 \mathrm{~min}\right)$ formed the kirenol. Based on the empirical formula for 15,16-dihydroxy ent-pimarane skeletons [21,24,25], the chemical shift of C-15 in kirenol was 76.5 ppm. Therefore, the structure of compound $\mathbf{3}$ was defined as 16-malonyl-2 $\alpha, 15 R, 19$-trihydroxypimar-8(14)ene (16- $O$-malonylkirenol).

Diterpenoids 1-3 were not previously described in the literature and were first isolated from Sigesbeckia pubescens.

\section{Conclusions}

Diterpenoids $\beta$-glucopyranosyl-18-acetoxy-16 $\alpha, 17$-dihydroxykauran-19-oate (1), 15 $O$-malonylkirenol (2), and 16-O-malonylkirenol (3) were isolated for the first time from the aerial parts of Sigesbeckia pubescens by extraction and preparative column chromatography. The structures of the isolated compounds were elucidated and proved by NMR spectroscopy and mass spectrometry. The diterpenoids 1-3 were new and not previously described in the literature.

\section{Funding}

This research received no external funding. 


\section{Acknowledgments}

This research has no acknowledgment.

\section{Conflicts of Interest}

The authors declared no potential conflict of interest.

\section{References}

1. Wang, R.; Chen, W.-H.; Shi, Y.-P. ent-Karane and ent-Pimarane Diterpenoids from Siegesbeckia pubescens. J. Nat. Prod. 2010, 73, 17-21, https://doi.org/10.1021/np9005579.

2. Yuan, Q.-Q.; Song, W.-B.; Wang, W.-Q.; Wei, J.-J.; Li, J.; Liu, Y. New ent-Kaurane and ent-Pimarane diterpenoids from Siegesbeckia pubescens. Phytochemistry Letters 2017, 21, 273-277, https://doi.org/10.1016/j.phytol.2017.07.003.

3. Xiong, J.; Ma, Y.; Xu, Y. Diterpenoids from Siegesbeckia pubescens. Phytochemistry 1992, 31, 917-921, https://doi.org/10.1016/0031-9422(92)80039-H.

4. Zhang, Q.R.; Zhong, Z.F.; Sang, W.; Xiong, W.; Tao, H.X.; Zhao, G.D.; Li, Z.X.; Tse, A.K.W; Hu, Y.J.; Yu, H. Comparative com-prehension on the antirheumatic Chinese herbal medicine Siegesbeckiae Herba: combined computational predictions and experimental investigations. J. Ethnopharmacol. 2019, 228, 200209, https://doi.org/10.1016/j.jep.2018.09.023.

5. Tao, H.X.; Zhao, G.D.; Linghu, K.G.; Xiong, W.; Sang, W.; Wang, Y.; Yu, H. Botany, traditional use, phytochemistry, pharmacology and toxicology of Sigesbeckie Herba (Xixiancao): a review. Phytochem. Rev. 2020, https://doi.org/10.1007/s11101-020-09714-4.

6. Linghu, K.G.; Zhao, G.D.; Xiong, W.; Sang, W.; Xiong, S.H.; Tse, A.K.W.; Hu, Y.; Bian, Z.; Wang, Y.; Yu, H. Comprehensive comparison on the anti-inflammatory effects of three species of Sigesbeckia plants based on NF-kB and MAPKs signal pathways in vitro. J. Ethnopharmacol. 2020, 250, 112530, https://doi.org/10.1016/j.jep.2019.112530.

7. Wang, Q.; Liang, Y.-Y.; Li, K.-W.; Li, Y.; Niu, F.-J.; Zhou, S.-J.; Wei, H.-C.; Zhou, C.-Z. Herba Siegesbeckiae: A review on its traditional uses, chemical, constituents, pharmacological activities and clinical studies. J. Ethnopharmacol. 2021, 275, 114117, https://doi.org/10.1016/j.jep.2021.114117.

8. Quan, P.; Jiao, B.; Shang, R.; Liu, C.; Fang, L. Alternative therapy of rheumatoid arthritis with a novel transdermal patch containing Siegesbeckiae Herba extract. J. Ethnopharmacol. 2021, 265, 113294, https://doi.org/10.106/j.jep.2020.113294.

9. Wu, J.Y.; Chan, Y.C.; Guo, H.; Chen, Y.J.; Liu, Y.X.; Yi, H.; Yu, Z.L. Twenty-four- week oral dosing toxicities of Herba Siegesbeckiae in rats. BMC Complement Med. Ther. 2020, 20, 341, https://10.1186/s12906020-03137-6.

10. Sun, Z.; Zhang, Y.; Zhou, H.; Xu, J.; Gu, Q. Diverse diterpenoids and sesquiterpenoids from Siegesbeckia pubescens and their activity against RANKL-induced osteoclastogenesis. Bioorganic Chemistry 2021, 107, 104537, https://doi.org/10.1016/j.bioorg.2020.104537.

11. Xu, F.; Hu, H.; Li, Y.; Ren, Y.; Zhao, H.; Huang, Q.; Wang, J. A. New ent-Pimarane-Type Diterpenoids Glycoside from Siegesbeckia pubescens. Rec. Nat. Prod. 2018, 12, 493-497, https://doi.org/10.25135/rnp.44.17.11.186.

12. Wang, J.; Xie, K.; Duan, H.; Wang, Y.; Ma, H.; Fu, H. Isolation and characterization of diterpene glycoside from Siegesbeckia pubescens. Bioorganic\&Medicinal Chemistry Letters 2017, 27, 1815-1819, https://doi.org/10.1016/j.bmcl.2017.02.051.

13. Li,Y.-S.; Zhang, J.; Tian, G.-H.; Shang, H.-C.; Tang, H.-B. Kirenol, darutoside and hesperidin contribute to the anti-inflammatory and analgesic activities of Siegesbeckia pubescens Makino by inhibiting COX-2 expression and inflammatory cell infiltration. J. Ethnopharmacol. 2021, 268,113547, https://doi.org/10.1016/j.jep.2020.113547.

14. Lin, Y.-Y.; Xia, H.; Xia, G.-Y.; Li, X.-Y.; Zhao, C.; Zhang, X.-T.; Lin, S.; Tian, G.-H. Effect of Siegesbeckiae Herba in treating chronic pain. China J. Chin. Mater. Med. 2020, 45, 1851-1858, https://doi.org/10.19540/j.cnki.cjcmm.20191016.501. 
15. Sun, Z.; Zhang, Y.; Zhou, H.; Xu, J.; Gu, Q. Diverse diterpenoids and sesquiterpenoids from Siegesbeckia pubescens and their activity against RANKL-induced osteoclastogenesis. Bioorg. Chem. 2020, 10, 104537, https://doi.org/10.1016/j.bioorg.2020.104537.

16. Nam, J.H. Vasodilatation effect of kirenol isolated from Sigesbeckia pubescens. Korean J. Polar. Res. 2020, 33, 467-475, https://10.7732/KJPR.2020.33.5.467.

17. Ren, J.; Yang, M.; Chen, J.; Ma, S.; Wang, N. Anti-inflammatory and wound healing potential of kirenol in diabetic rats through the suppression of inflammatory markers and matrix metalloproteinase expressions. Biomed. Pharmacother 2020, 129, 110475, https://doi.org/10.1016/j.biopha.2020.110475.

18. Liu, W.; Li, Y.; Li, C. Kirenol exhibits the protective role against N-Methyl-N-Nitrosourea-induced gastric cancer in rats via modulating the oxidative stress and inflammatory markers. J. Environ. Pathol. Toxicol. Oncol. 2020, 39,345-355, https://doi.org/10.1615/JEnvironPatholToxicoOncol.2020035475.

19. Teng, T.; Xu, S.; Chen, F.; Li, X.; Gao, L.; Ye, Y. Research Progress in Chemical Constituents and Pharmacological Activities of Siegesbeckie Herba. Chin. J. Mod. Appl. Pharm. 2015, 32, 250-260, https://doi.org/10.12677/TCM.2021.101002.

20. Gao, H.; Li, P.; Li, D.; Du, X. Studies on chemical constituents of Siegesbeckia pubescens. Chinese traditional and Herbal drugs 2002, 6, 495-496.

21. Wang, J.; Duan, H.Q.; Wang, Y.; Pan, B.; Gao, C.; Gai, C.; Wu, Q.; Fu, H. Ent-Strobane and Ent-Pimarane Diterpenoids from Siegesbeckia pubescens. J. Nat. Prod. 2017, 80, 19-29, https://doi.org/10.1021/acs.jnatprod.6b00150.

22. Shim, S.-Y.; Lee, Y.E., Lee, M. Antioxidant compounds, kirenol and methyl ent-16a,17-dihydroxy-kauran19-oate Bioactivity-Guided isolated from Siegesbeckia glabrescens attenuates MITF-mediated melanogenesis via inhibition of intracellular ROS production. Molecules 2021, 26, 1940, https://doi.org/10.3390/MOLECULES26071940.

23. Ibrahim, S.R.M.; Altyar, A.E.; Sindi, I.A.; El-Agamy, D.S.; Abdallah, H.M.; Mohamed, S.G.A.; Mohamed, G.A. Kirenol: A promising bioactive metabolite from Siegesbeckia species: A detailed review. J. Ethnopharmacol. 2021, 281, 114552, https://10.1016/j.jep.2021.114552.

24. Giang, P.M.; Son, P.T.; Otsuka, H. ent-pimarane-type diterpenoids from Siegesbeckia orientalis L. Chem. Pharm. Bull. 2005, 53, 232-234, https://doi.org/10.1248/cpb.53.232.

25. Gao, X.; Rong, Z.; Long, G.; Hu, G.; Yan, T.; Li, N.; Jia, J.; Wang, A. ent-Pimarane diterpenoids from Siegesbeckia glabrescens with anti-inflammatory activity. Bioorg. Chem. 2020, 99, 103854, https://doi.org/10.1016/j.bioorg.2020.103854. 